\title{
Probabilistic sensitivity analysis of offshore wind turbines using a transformed Kullback-Leibler divergence
}

\author{
Rui Teixeira, Alan O'Connor, Maria Nogal \\ Department of Civil, Structural and Enviornmental Engineering, College Green, Dublin 2 \\ rteixeir@tcd.ie,oconnoaj@tcd.ie,nogalm@tcd.ie
}

\begin{abstract}
Characterizing uncertainty in complex systems is steadily growing as a topic of interest. One of the efficient ways to characterize a complex system is achieved by probabilistic sensitivity analysis. In the context of performing it, there are a limited number of methods to quantify the change of the output to its full probabilistic extent. Moreover, in some engineering applications, such as reliability analysis, some established indicators of sensitivity do not fit the best interest of the analysis.

This is the case of Kullback-Leibler divergence. Despite applied for probabilistic sensitivity analysis, it has limited interest in certain circumstances. A transformation of this indicator of entropy between two distributions is proposed in the present work. This transformation is used to establish a complementary indicator that is more perceptive, and more efficient for reliability analysis. This new function is applied for the global sensitivity analysis of an offshore wind turbine on a monopile foundation.

Results show that, for engineering problems as the one presented, the usage of this transformed indicator produces intuitive results. It allows the efficient identification of relevant states of operation as well as the most influent variables in the design of experiments, resulting in better comprehension of system's behaviour and operational risks.
\end{abstract}

Keywords: Operational risk, Kullback-Leibler divergence, Probabilistic Sensitivity Analysis, Offshore Wind Energy, Structural Fatigue, Design of experiments

\section{Introduction}

Characterizing uncertainty is steadily increasing as a global concern in multiple fields of knowledge. In the case of engineering systems, uncertainty characterization is particularly important when dealing with complex systems that are affected by multiples sources of uncertainty. For engineering applications the 
designer needs to be conscious that it is not sufficient to approach the design of a system, even if choosing to over-design, without any awareness of the uncertainties that affect it. He or she needs to find strategies to have a comprehensive understanding of the system's response.

Saltelli et al. (2008) states that uncertainty quantification and sensitivity analysis, in its more broad understanding of identifying the most relevant variables in the Design of Experiments (DoE), run in tandem. One of the most adequate ways to characterize a system is by conducting a sensitivity analysis, which can be attained by applying efficient and established Global Sensitivity Analysis (GSA) techniques.

A GSA enables the designer to increase his or her awareness about the operation of a certain system. Yet, alone it does not characterize its probabilistic behaviour. If the goal is to obtain a probabilistic description of the results, a probabilistic GSA (P-GSA) is demanded.

One of the strategies to solve the problem of P-GSA for complex systems is to build importance measures that effectively quantify the sensitivity of the output variable in regard of changes in the input field (Caniou, 2012).

The following work proposes an approach for P-GSA, applied to an OWT, that involves: defining a complementary importance measure for P-GSA; characterizing this importance measure; and applying it to create a global map of sensitivity results.

The measure of sensitivity used is the Kullback-Leibler (KL) divergence, a measure of entropy between statistical distributions. A transformation is proposed in order to improve the understanding of the P-GSA field for the studied physical variable.

To tackle the proposed objective the present paper is organized as follows: Section 2 introduces the theoretical concepts behind GSA and presents some reference works developed in the past; Section 3 discusses the considerations needed to develop GSA for a long-term event such as structural fatigue damage; Section 4 proposes a novel approach to the P-GSA, discusses it and presents the results for an OWT tower; finally, Section 5 draws the main conclusions of the work developed.

\section{Probabilistic global sensitivity analysis for complex models}

Two main challenges are encountered when a P-GSA analysis is pursued. The first, relates to the difficulty of identifying the range in the space of variables where the sensitivity must be assessed. The second relates to the difficulty, when dealing with a high dimensional space, to not seriously compromise the assessment efficiency by using an unreasonably large design space.

For complex problems, a non-deterministic output response may also occur. It is the case when a single set of deterministic inputs generates a random output. In such cases, the focus of the sensitivity analysis needs to consider the complete probabilistic behavior of the output.

A degree of quantification of uncertainty can be achieved with variance-based methods such as Efron and Stein (1981) or Sobol (1993). The works by Rabitz 
et al. (1999), Saltelli et al. (2000) or Sobol (2001) created a consolidated basis for the application of variance based methods in sensitivity analysis, which resulted in extensive literature that characterizes sensitivity using variance-based methods, e.g. (Saltelli et al., 2010; Zhang et al., 2015; Yun et al., 2017).

Nonetheless, Borgonovo (2007) comprehensively discussed the limitations of the sensitivity analysis based on the analysis of variance. To analyse complex systems, such an OWT, the major interest is to infer on the entire distribution of the output, which implies the need to develop a GSA that considers it. Other statistical moments are of interest in such cases. However, unlike the variancebased methods, these do not add up to the unit. As a result, their application is case-specific.

Many authors identified the necessity to quantify the disparity between two distributions and proposed measures for it, such as Kullback and Leibler (1951) or Borgonovo (2007). Other established alternatives are presented in Rachev (1991).

Assuming two Probability Density Function (PDF) of an output variable $Y(x), f_{Y}(x)$ and $f_{Y^{*}}(x)$. For both, $f_{Y}(x)$ and $f_{Y^{*}}(x)$, the Kullback-Leibler (KL) measure of divergence assesses the relative entropy between the two PDF and is defined as

$$
D_{K L}\left(f_{Y}(x) \| f_{Y^{*}}(x)\right)=\int_{x} f_{Y}(x) \ln \frac{f_{Y}(x)}{f_{Y^{*}}(x)} d x,
$$

taking the following form if $f_{Y}(x)$ and $f_{Y^{*}}(x)$ are univariate normal distributions with mean $\mu_{f}$ and $\mu_{f^{*}}$, and standard deviation $\sigma_{f}$ and $\sigma_{f^{*}}$ respectively.

$$
\begin{aligned}
& D_{K L}\left(f_{Y}(x) \| f_{Y^{*}}(x)\right)=\ln \left(\frac{\sigma_{f}^{*}}{\sigma_{f}}\right)+\frac{\sigma_{f}^{2}+\left(\mu_{f}-\mu_{f^{*}}\right)^{2}}{2 \sigma_{f^{*}}^{2}}-\frac{1}{2} \\
& \text { with }, \mu_{f}, \mu_{f}^{*} \in \mathbb{R} ; \sigma_{f}, \sigma_{f}^{*} \in \mathbb{R}^{+}
\end{aligned}
$$

$D_{K L}$ is non-monotonic always positive. Its function is symmetric in relation to changes in $\mu$, non-symmetric in relation to variations in $\sigma$, and has a vertical asymptotic behaviour when $\sigma_{f^{*}} \rightarrow 0$.

The divergence is a non-symmetric measure of distance between two distributions. The symmetrized divergence $(J)$ was introduced in Kullback (1997). In the case of $J$, the divergence is analysed both ways between $f_{Y}(x)$ and $f_{Y^{*}}(x)$.

The idea of using the divergence to analyse the sensitivity emerged first in Park and Ahn (1994), where the KL discriminator is used to assess and compare probabilistic sensitivity results. Liu et al. (2006) applies the same measure justifying its application by its capability of enclosing more uncertainty information. Balesdent et al. (2013) uses the divergence as a cross-entropy sensitivity search indicator in the DoE. Hoseyni et al. (2015) applies it to characterize sensitivity in a finite mixture model analysis. Greegar and Manohar (2016) uses the KL in a comparison of sensitivity indices, using also the Hellinger, Wassertein (Kantorovic) and the $I^{2}$ metrics.

Regardless of the indicator applied, in the sensitivity analysis of non-linear problems, whose evaluation commonly requires high computational time, it is 
not feasible to cover the design space and set the design of experiments (DoE) without an established criteria. Full insight on the importance of a balanced sampling approach to GSA is discussed in Saltelli et al. (2008).

Random sampling, importance sampling or latin hypercube sampling (LHS) are examples of widely used techniques. In particular, LHS is efficient for resource consuming models as it allows covering efficiently the DoE with a relatively small sample size. A review of the main advantages of the LHS for analysis of multi-scale systems with discussion of its applicability for sensitivity analysis and usefulness to map the DoE can be found in Helton and Davis (2003). In the specific case of reliability problems, Olsson et al. (2003) discusses its interest as a sampling method. Even considering that LHS introduces a more balanced approach to map the DoE, for complex problems the computational effort can remain quite demanding. Additionally, it is not a good approach when focusing on extreme values, given the low probability cumulated under the tails. Improvements of the LHS use specific criteria to balance the way the points are picked in the probability distributions (Viana et al., 2010).

\section{Definition of an importance measure}

\subsection{Fatigue damage indicator}

Structural fatigue is a long-term cumulative process. Fatigue analysis of OWT components involves running multiple time-domain simulations, conducting a rainflow counting of the load amplitudes, means and cycles, setting the short-term distribution of loads, extrapolating for long-term occurrences and finally assessing the life-time damage (IEC, 2005).

A sensitivity analysis of an OWT component to fatigue requires the definition of a design "damage indicator" $(D i)$. The definition of a representative $D i$ is a wide challenge. Similarly to how different related approaches can be found to assess fatigue, stress based, strain based and fracture mechanics, the same occurs in the definition of $D i$. Mesmacque et al. (2005) highlight the difficulty of defining a representative $D i$. There are many different approaches to assess fatigue (Fatemi and Yang, 1998), and depending on the approach, different indicators can be defined. It is important, when discussing fatigue in sensitivity analysis, to clearly define what indicator is used to compare the results.

For the proposed P-GSA the short-term stress damage rate $\left(D_{S H}\right)$ induced in the tower is proposed as a $D i$ for comparison of the results. $D_{S H}$ is assessed by calculating the load ranges and cycles in a reference time $t$ using the PalmgrenMiner rule, Equation 3.

$$
D_{S H}=\sum_{S_{t_{i}}}^{t_{n}} \frac{n_{S_{t_{i}}}}{N_{S_{t_{i}}}}
$$

where $N_{S_{t_{i}}}$ is the maximum allowed cycles at the stress amplitude $S_{t_{i}}$ according to a pre-defined stress-cycle curve and $n_{S_{t_{i}}}$ is the recorded number of stress cycles, with $t_{n}$ as the number of loads recorded in the reference time $t$ with $t_{i}=1, \ldots, t_{n}$. The stress-based approach is the design technique recommended 
by IEC (2005). Nevertheless, the P-GSA methodology here proposed can be applied regardless of the damage indicator used.

Double slope stress-cycle curve defined by DNV (2014) is applied in the representative example. A 5MW turbine on a monopile foundation is considered due to its baseline character (Jonkman et al., 2009). The damage rates were validated with Hayman (2012).

$D_{S H}$ in an OWT tower depends on a set of parameters $\boldsymbol{\Theta}=\left[\theta_{1}, \theta_{2}, \ldots, \theta_{n}\right]$ that influences the OWT tower loading. $\Theta$ is expressed as a row vector of length $n$ containing $\theta_{i}$ random variables that can take multiple values. In the case of $D_{S H}(\boldsymbol{\Theta})$ every singular combination of $\theta_{i}$ parameters will originate a conditional output probability distribution. In the case of fatigue analysis, there is a requirement to work with the entire output distribution as a quantifier of uncertainty. The mean value has significant importance in the problem of fatigue (Sutherland, 1999).

When developing a P-GSA analysis it is interesting to identify which distribution $D_{S H}\left(\boldsymbol{\Theta} \mid \Theta_{i}\right)$ follows, with $\boldsymbol{\Theta}_{i}$ as a generic $i$ combination of $\theta_{i}$ values.

\subsection{P-GSA Design of Experiments}

Fourteen environmental random variables were considered, $n=14$, in the representative P-GSA developed. The studied variables are presented in Table 1.

To cover the DoE, real data presented in Teixeira et al. (2018) from the M6 buoy was used to ensure a realistic approximation to the DoE environmental variables. The distributions for each input variable used in the modeling are shown in Table 1. In the cases recorded data were not available a reference study was applied to characterize the reference intervals for the variables considered, such as the wind profile and terrain uncertainty (Veldkamp, 2006; Jonkman, 2009; Toft et al., 2016). Türk and Emeis (2010) data was applied to extend the $I$ dataset for the height considered.

The corresponding probability papers were used to confirm the presented selection of distributions.

A Latin Hypercube Sampling (LHS) was implemented for the P-GSA procedure. It is important to understand that some of the variables are correlated and this influences the implementation of the LHS. Spearman correlation coefficients between the different variables are presented in Table 1 . The coefficients not presented in the table, or presented a "very weak" correlation, or were assumed to be independent.

The correlation that exists between the $\theta$ variables considered increases the complexity of applying the LHS. The methodology introduced in Iman and Conover (1982) to sample correlated variables is then applied to account for the correlation between the considered variables. Two lower triangular matrices $P$ and $Q$ are obtained using the Cholesky factorization of the Spearman correlation matrices with target correlation $C=P P^{\prime}$ and the correlation matrix of the 
Table 1: Random variables considered in the LHS and respective distributions

\begin{tabular}{|c|c|c|c|c|}
\hline Variable & & PDF & Parameters & Corr $\left(r_{s}\right)$ (Spearman $)$ \\
\hline$U(m / s)$ & Wind velocity & Weibull & $(9.7,2.5)$ & $\begin{array}{l}r_{s}\left(U, H_{s}\right) \\
0.66 ; r_{s}\left(U, T_{p}\right)=0.27\end{array}$ \\
\hline$H_{s}(m)$ & Significant Wave Height & Weibull & $(3.9,2.1)$ & $\begin{array}{l}r_{s}\left(U, H_{s}\right) \\
0.66 ; r_{s}\left(H_{s}, T_{p}\right)=0.79\end{array}$ \\
\hline$T_{p}(s)$ & Peak Period & Rayleigh & $(7.3)$ & $\begin{array}{l}r_{s}\left(U, T_{p}\right) \\
0.27 ; r_{s}\left(H_{s}, T_{p}\right)=0.79\end{array}$ \\
\hline$I(\%)$ & Turbulence Intensity & Normal & $(5.7,2.9)$ & $r_{s}(U, I)=-0.30$ \\
\hline$\Phi_{w}(0)$ & Wind direction & Uniform & {$[-15,15]$} & - \\
\hline$\omega_{w}(\circ)$ & Incident wave direction & Uniform & {$[-15,15]$} & - \\
\hline$\rho_{\text {air }}\left(\mathrm{kg} / \mathrm{m}^{3}\right)$ & Air density & Normal & $(1.24,0.08)$ & - \\
\hline$\rho_{\text {water }}\left(\mathrm{kg} / \mathrm{m}^{3}\right)$ & Water density & Normal & $(1027,0.5)$ & - \\
\hline$\alpha$ & Shear parameter & Normal & $(0.18,0.02)$ & - \\
\hline$z_{0}$ & Surface roughness length & Normal & $(0.03,0.003)$ & - \\
\hline $\mathrm{Ri}$ & Richardson gradient & Uniform & {$[0.03,0.05]$} & - \\
\hline$\gamma$ & $\begin{array}{l}\text { Jonswap spectra param- } \\
\text { eter }\end{array}$ & Uniform & {$[3.3,4.8]$} & - \\
\hline$U_{C}(m / s)$ & Current velocity & Uniform & {$[0,1]$} & - \\
\hline MSL (m) & Mean sea Water level & Uniform & {$[2.5,-2.5]$} & - \\
\hline
\end{tabular}

independently assessed LHS $\left(T=Q Q^{\prime}\right)$.

$$
\begin{gathered}
R_{B}^{*}=R S^{\prime} \text { with } \\
S=P Q^{-1}
\end{gathered}
$$

$R^{*}$, transformed of $R$, has correlation matrix equal to the imposed $C$ and preserves the initial univariate distributions.

The $\phi_{p}(D)$ distance criteria introduced in Morris and Mitchell (1995) is applied to validate the correlated and non-correlated samples and optimize different DoEs.

Selecting an ideal sample size to perform a GSA is challenging. A minimum sample size of $n+1$ is usually assumed for LHS, but other recommendations are found on the adequate sample size. Iman and Helton (1985) have defined a sample size of $4 / 3 n$ as satisfactory to cover the DoE. Manache and Melching (2008) refers that this measure was compared with the value of $3 n$ to test its robustness and positive results of convergence were achieved. Nevertheless many authors do not follow strictly the rule presented in Iman and Helton (1985) and select the sample size which they believe that will produce efficient results, or for which they are investigating the adequacy. Examples can be identified in Stein (1987) where a sample size $s=100$ is used for $n=6$ while Helton et al. (2005) uses the same sample size for a $n=31$.

A LHS of size $s=30$ is applied in the example presented. The criteria to select $s$, additionally to requiring a minimum reference $s$ within the references presented for its selection, was to achieve adequate resolution in the variable of the space that is expected to cover a wider physical variation in absolute values, $U$. Only power production (between the cut-in and cut-out wind speeds) is evaluated due to its major importance for fatigue (Veldkamp, 2006). 


\subsection{Distribution of $D_{S H}$}

Many natural processes are Gaussian distributed, but this is not always the case. For the case of fatigue it is commonly assumed that under constant or random loading a normal or log-normal distribution will describe accurately the variability of the fatigue damage (Wu et al., 1997; Wirsching and Chen, 1988).

To infer on the variability of $D_{S H}(\boldsymbol{\Theta})$ the statistical output was studied by using probability papers to compare the likelihood of different statistical distributions. The results showed that the lognormal distribution appears to approximate well the statistical behaviour of $D_{S H}(\boldsymbol{\Theta})$, Figure 1. The fact that
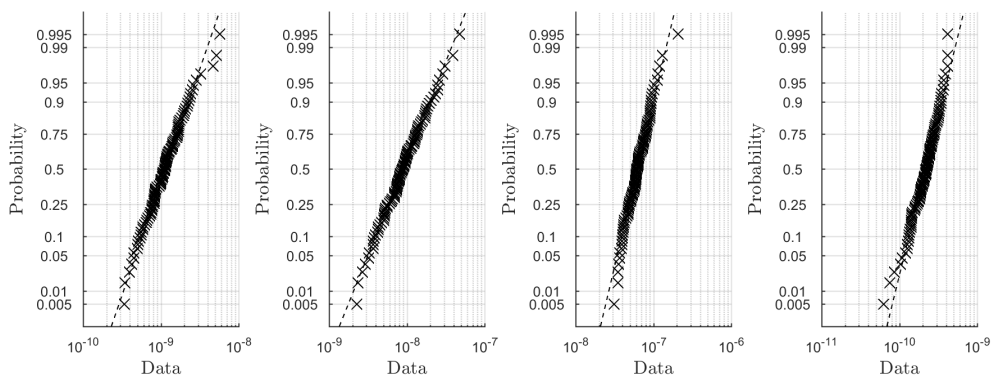

Figure 1: Log-normal probability papers of $D_{S H}(\boldsymbol{\Theta})$ for $4 \boldsymbol{\Theta}$ combinations.

$D_{S H}(\boldsymbol{\Theta})$ is well represented by a lognormal distribution may be related to the requirement for $D_{S H}$ to be always positive.

It is known that the logarithm of a lognormal distributed variable follows a normal distribution. Since $D_{S H}$

$$
D_{S H}(\boldsymbol{\Theta}) \sim \ln \mathcal{N}\left(\mu, \sigma^{2}\right) .
$$

then,

$$
\ln \left(D_{S H}(\boldsymbol{\Theta})\right) \sim \mathcal{N}\left(\mu, \sigma^{2}\right) .
$$

$D_{S H}(\Theta)$ is assumed to follow a $\log$-normal distribution $\forall \Theta$. This consideration is important since $D_{K L}(\boldsymbol{\Theta})$ can be represented in a closed form dependent on continuous distributions.

100 evaluations were applied for probability characterization in order to estimate the uncertain behavior of the tower fatigue.

\section{Global Sensitivity Analysis of $D_{S H}$}

\subsection{Transformed sensitivity indicator.}

When developing a sensitivity analysis to characterize an engineering design variable, as the one studied in this example, it is important to produce perceptive results. This means that the results should be intuitive for the designer and enable her or him to easily draw conclusions. 
For some physical quantities the interest is not only to characterize how far one distribution moves from one another in terms of probability, but also to characterize the physical process in regard of its practical effect. An increase in the standard deviation may contribute to a decrease in the statistical distance to a reference distribution, however in terms of practical effect (e.g. for the reliability problem) this increase may represent a more severe operational condition and therefore should be characterized in terms of sensitivity as that.

$D_{K L}$ characterizes the entropy between two distributions. When using it to characterize the distance between two distributions, it is difficult to infer on the relative location of the sensitivity point on the probabilistic map without further operations.

Consider $\mathbb{R}_{\mu^{+}}, \mathbb{R}_{\sigma^{+}}^{+}$and $\mathbb{R}_{\mu^{-}}, \mathbb{R}_{\sigma^{-}}^{+}$as separate domains of the KL function where the change in mean and standard deviation are positive and negative respectively.

While the KL function is symmetric between its both domains, $\mu^{+}$and $\mu^{-}$, the same is not verified in the domains $\sigma^{+}$and $\sigma^{-}$. Similarly, the KL function is monotonic increasing in both domains of $\mathbb{R}_{\mu}$ and $\mathbb{R}_{\sigma^{-}}^{+}$, but non-monotonic in $\mathbb{R}_{\sigma^{+}}^{+}$.

For engineering application purposes it may be of interest to have a complementary KL as a symmetric and monotonic function in all domains in order to have a more perceptive description of the P-GSA. Despite applied in previous works, its original function shape has limited interest for mapping G-PSA results in practical examples, such as reliability analysis.

A transformation to the KL function is proposed in order to enable it to be an efficient indicator of G-PSA. This transformed function adds complementary information on sensitivity to $D_{K L}$ that is of interest for practical applications. To ensure a monotonic and symmetric behaviour in all the domains identified, the KL function should be modified in $\mathbb{R}_{\sigma^{+}}^{+}$using the following transformations $g_{I}$ and $g_{I I}$,

$$
\begin{gathered}
g_{I}: D_{K L} \rightarrow D_{K L}^{I}, \quad \forall \mu_{f} \neq \mu_{f^{*}} \cap \sigma_{f^{*}} \in \mathbb{R}_{\sigma^{+}}^{+} \\
g_{I}=\left\{\begin{array}{lc}
2 \frac{D_{K L}\left(\sigma_{f^{*}}=\sigma_{f}\right)}{D_{K L}\left(\sigma_{f^{*}}\right)}-1 & \sigma_{f}>\sigma_{f^{*}} \geq \sigma_{f_{m i n}^{*}} \\
2 \frac{\left|D_{K L}\left(\sigma_{f^{*}}=\sigma_{f}\right)-D_{K L}\left(\sigma_{f^{*}}=\sigma_{f_{m i n}^{*}}\right)\right|}{D_{K L}\left(\sigma_{f^{*}}\right)}+1 & \sigma_{f^{*}}>\sigma_{f_{m i n}^{*}}
\end{array}\right.
\end{gathered}
$$

with $\sigma_{f_{m i n}^{*}}$ being the value of $\sigma_{f}^{*}$ for the respective $\mu_{f}$ and $\mu_{f}^{*}$ for which the $D_{K L}$ is minimum. Then $g_{I I}$ is applied accordingly to,

$$
\begin{array}{cc}
g_{I I}: D_{K L}^{I} \rightarrow D_{K L}^{I I}, \quad \forall \mu_{f}, \mu_{f^{*}} \cap \sigma_{f^{*}} \in \mathbb{R}_{\sigma^{-}}^{+} \\
g_{I I}=\frac{D_{K L}^{I}\left(\sigma_{f}+\left|\sigma_{f}-\sigma_{f^{*}}\right|\right)}{D_{K L}\left(\sigma_{f^{*}}\right)} & 0<\sigma_{f^{*}}<\sigma_{f}
\end{array}
$$

along the remaining domains, the partial transformations $g_{I}$ and $g_{I I}$ will have 
the value of one unit as no transformation is expected,

$$
\begin{array}{ll}
g_{I}=1, & \forall \mu_{f}, \mu_{f^{*}} \cap\left(\sigma_{f^{*}} \in \mathbb{R}_{\sigma^{-}}^{+} \cup \sigma_{f^{*}}=\sigma_{f}\right), \\
g_{I I}=1 & \forall \mu_{f}, \mu_{f^{*}} \cap\left(\sigma_{f^{*}} \in \mathbb{R}_{\sigma^{+}}^{+} \cup \sigma_{f^{*}}=\sigma_{f}\right),
\end{array}
$$

This transformation could be implemented through a single transformation using

$$
\left[g(I-I I)=g_{I} g_{I I}\right]: D_{K L} \rightarrow D_{K L}^{I I}
$$

$g(I-I I)$ is a global transformation function while $g_{I}$ and $g_{I I}$ are local transformations functions as presented in Equations (6 - 9). The division in a two step function intends to facilitate the understanding of the proposed implementation.

Figure 2 presents the original KL function, transformation, and the transformed functions for both the cases where $\mu_{f}=\mu_{f^{*}}$ and $\mu_{f} \neq \mu_{f^{*}}$. The second is representative of both $\mathbb{R}_{\mu^{-}}$and $\mathbb{R}_{\mu^{+}}$due to the symmetry of the KL function regarding differences in $\mu$.

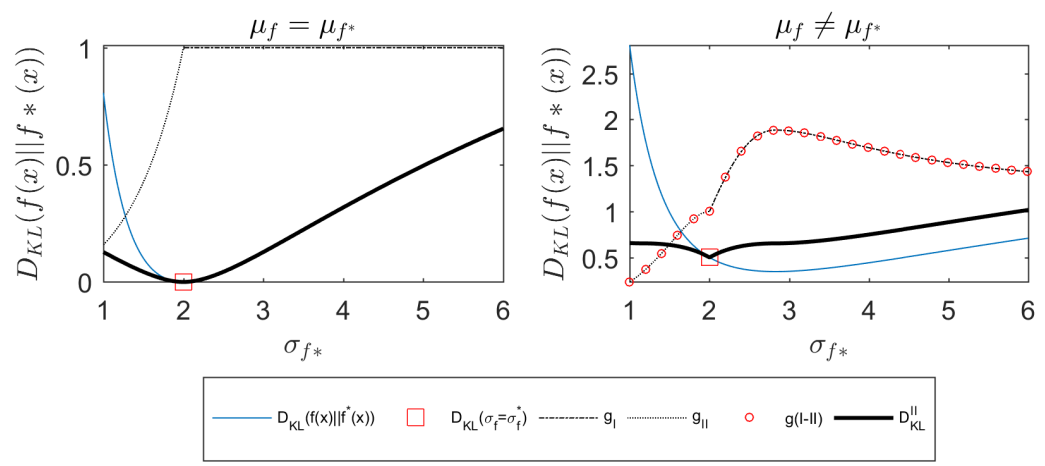

Figure 2: Example of the $D_{K L}$ function for the cases when $\mu_{f}=\mu_{f}$ and $\mu_{f} \neq \mu_{f^{*}}$ for a distribution with $\mu_{f}=10$ and $\sigma_{f}=2$.

The shape of the $D_{K L}$ function can be identified in Figure 2. KL is not symmetric in regard of changes in $\sigma$. When $\mu_{f}=\mu_{f}^{*}$ the minimum of $D_{K L}$ occurs when $\sigma_{f}=\sigma_{f^{*}}$, therefore in this case $g(I-I I)=g_{I I}$. When $\mu_{f} \neq \mu_{f}^{*}$, due to the shift caused by the difference in the means, a similar minimum for $\sigma_{f}=\sigma_{f^{*}}$ does not occur. $g_{I}$ main purpose is then to guarantee that the local minimum for $\forall \mu_{f} \neq \mu_{f^{*}}$ occurs at $\sigma_{f}=\sigma_{f^{*}}$

Two examples of application of $D_{K L}^{I I}$ are presented in Figure 3. The first (a) presents a univariate data set where the comparative distribution $f^{*}$ is progressively modified in relation to a reference distribution $f$. For the sake of illustration, seven sets with different $\mu$ and $\sigma$ values are shown. For each set, two $\sigma$ values have been considered, i.e., the reference one and one selected randomly. Even considering that the $f^{*}$ is not progressively more distant from $f$ as given by $D_{K L}$, its statistical moments are. The results for the discrimination are presented to the right. With the single results for $D_{K L}$ it is difficult in terms 
of sensitivity to identify changes in $\mu_{f^{*}}$ and in $\sigma_{f^{*}}$. This effect is significant as the ratio between $\mu_{f}$ and $\mu_{f^{*}}$ increases. When this ratio is 1.5 , it can be seen that an increase in the $\sigma_{f^{*}}$ results in a $D_{K L}$ smaller than when this same ratio is approximately 1.4 and the value of $\sigma_{f^{*}}$ closer to $\sigma_{f}$. On the other hand, $D_{K L}^{I I}$ is strictly increasing as the difference between the $f^{*}$ and $f$ moments increases. When conducting a sensitivity analysis this is of interest in order to produce perceptive results and should complement the analysis with $D_{K L}$. When the changes in the moments are negative, the $D_{K L}$ indicator increases rapidly while $D_{K L}^{I I}$ increases at the same rate of the symmetric positive changes. The second example (b) presents a bi-variate set of data that characterizes a phenomena where the changing behaviour of $\mu_{f^{*}}$ is mainly induced by the modification in $x$, and the change in $\sigma_{f^{*}}$ is ruled by the $y$ variable accordingly to the figure presented on the left. $x$ and $y$ are independent and can take any value in the field $(x, y), \mu_{f^{*}}$ and $\sigma_{f^{*}}$ are constrained to the values taken by $x$ and $y . D_{K L}$ maxima occurs for the points where the difference in $\mu$ is maxima but there is no change in $\sigma . D_{K L}^{I I}$ maxima occurs for the points where the change is maxima for both statistical moments. This example shows that, when using $D_{K L}$ as an indicator, if no further information is known about the statistical distributions it is difficult to identify accurately what are the probabilistic sensitivity results, while $D_{K L}^{I I}$ is able to complement this analysis accurately. When the difference in the $\mu$ and $\sigma$ are maxima, $D_{K L}$ takes the same value than when the $\mu_{f^{*}} / \mu_{f}$ is 1.5 with no change in $\sigma$. For some physical processes, such as loading, the combination of two maxima is expected to be more critical. In particular, for reliability calculations, the point where both maximums occurs is the most dangerous operational point because, with the same $\mu$, more extreme values may be experienced, or, in case of stress-cycle fatigue, the uncertainty in the mean estimation is larger. 

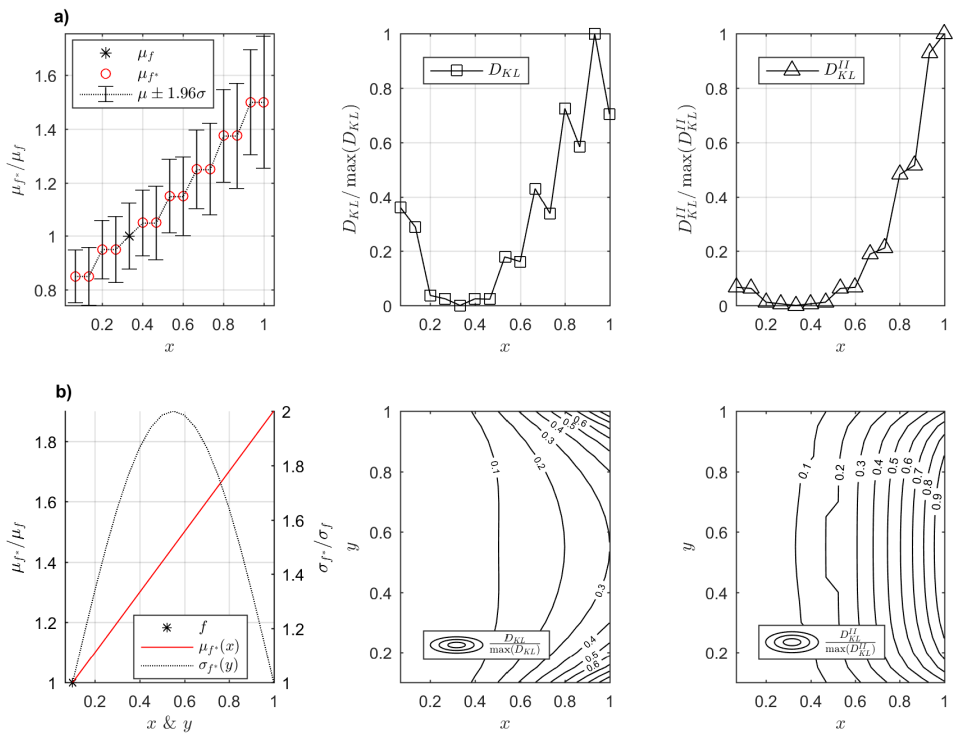

Figure 3: Example of implementation of KL divergence in the context of the P-GSA. a) represents a univariate set of data where both the first statistical moments are progressively increased and both $D_{K L}$ and $D_{K L}^{I I}$ are calculated to infer on the G-PSA. b) represents a set of data where the sensitivities of $\mu$ and $\sigma$ are mainly influence by the parameters $x$ and $y$, respectively.

While the interest of $D_{K L}^{I I}$ may not be so evident in some fields of knowledge, such as the ones that address the need to produce probability distributions that are far from each other; in reliability engineering, it is of relevance to produce more perceptive sensitivity results. It is possible to infer that, for P-GSA in a reliability context, $D_{K L}^{I I}$ enables a more perceptive description of operational risk, being able to complement the analysis with $D_{K L}$.

In order to have a sensitivity analysis, a reference point is demanded. Application of the transformation proposed requires only the characterization of the reference distribution in order to characterize the transformed function. This allows the characterization of the $D_{K L}^{I I}$ in a single step at the beginning of the calculations. Since the respective values for both indicators are defined, further calculations may be performed only for $D_{K L}$ or $D_{K L}^{I I}$, and interpolated from these. The numerical complexity of the sensitivity calculations may be reduced with this approach, however, its efficiency should be weighted. It may be of interest in terms of efficiency only if a very large number of points need to be evaluated, e.g. health monitoring applications. In these cases, usage of a reference to establish a reference map is of interest. If numerical efficiency becomes a limitation, it can be also improved with the application of approximations, such as probability function fits or coarse integration steps. For both alternatives, it is possible to characterize the uncertainty in the results and refine them if 
required. Therefore, the approach to the P-GSA computation, its accuracy, and its numerical efficiency is expected to be case-specific.

If further information is needed about $D_{K L}^{I I}$ the local derivatives can be used to estimate the positions in the indicator space. $D_{K L}^{I I}$ may be represented in its symmetrized form (Kullback, 1997) if a symmetric measure of sensitivity is required. It is noted that the transformed function does not obey to the triangle inequality in any of the cases. This same property is not respected by the $D_{K L}$ or $J$, and hence not by the transformed function.

\subsection{G-PSA Results}

The LHS generated for the set of parameters $\Theta$ is presented in Table 2. The first two statistical moments and two results for $D_{K L}$ and $D_{K L}^{I I}$ are also presented in the last six columns of the table.

In the current example two reference operation points were used to compute the G-PSA field, the hypothetical more favourable state in terms of reliability ${ }^{1}$ that could be characterized with the data generated and the real less damaging state that was recorded $(\Theta=1$ in Table 2$)$. The hypothetical less damaging state is characterized by the combination of the minimum $\mu$ and $\sigma$ (highlighted in bold in Table 2) possible in the data set considered. This hypothetical state is defined with the intent of having a progressively increasing measure of sensitivity that is associated with the expected reliability for the different points of operations considered. This is an interesting reference state to use in the analysis due to its capability of producing more perceptive and practical results.

For both the $D_{K L}$ results, the change in the indicator is minimal. The lower weight given to changes in the standard deviation can be identified by the similarity between both $D_{K L}$ values. Examples where this indicator does not produce perceptive results in the researched context can be found in multiple examples, such as $\boldsymbol{\Theta} 8,13$, or 22 when comparing with the cases of 10,14 , and 24 , respectively.

When considering $D_{K L}^{I I}, \boldsymbol{\Theta} 18$ and 22 are expected to be similar in damaging the OWT (similar values of $D_{S H}$ ). This is identified when considering the minimum values of $\mu$ and $\sigma$ as a reference point, however, the same does not happen when considering $\Theta=1$ as a reference point. This occurs due to the proximity of $\sigma$ between $\boldsymbol{\Theta}=1$ and 22 when comparing with $\boldsymbol{\Theta}=18$. As $D_{K L}^{I I}$ reflects positive changes in $\sigma$ additionally to $\mu$, the difference in the divergence between both points of operation increases while they are very likely to be similarly demanding regarding the structural fatigue of the tower component. With this example it is possible to understand that, in addition to using a perceptive measure of G-PSA, it is also of major importance when building a $D_{K L}^{I I}$ for practical problems to define a reference point that enables the most perceptive results considering the problem analysed. In a design basis, this would help the designer to achieve more comprehensive results.

\footnotetext{
${ }^{1}$ less reliability risk is associated with smaller probability of experiencing large short-term mean fatigue damage rate
} 


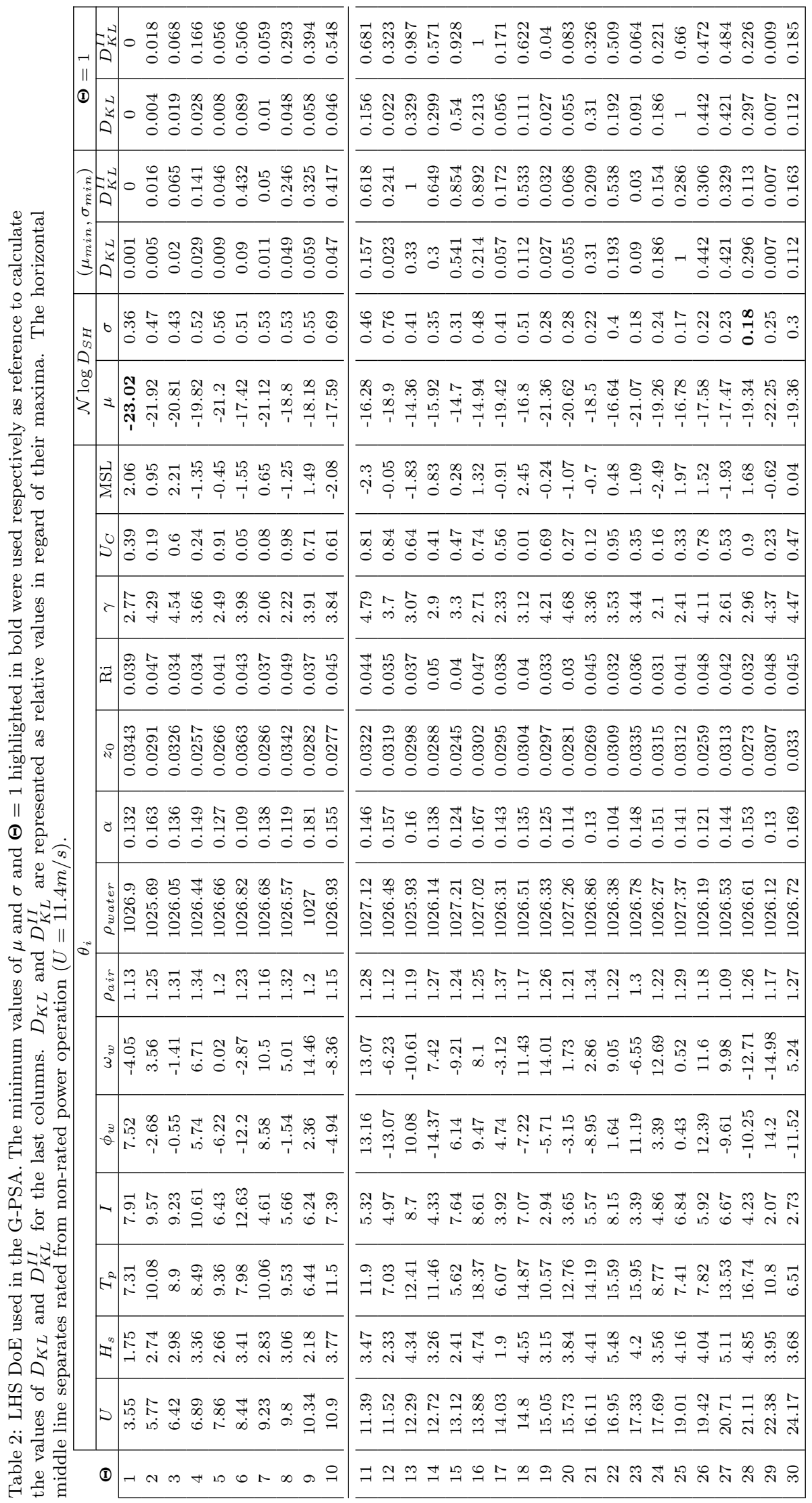


Figure 4 shows the variation of $D_{K L}^{I I}$ with the univariate change of each $\theta_{i}$ variable for the case where an hypothetical minimum state of operation is used as reference. The four vertical lines represent, in the order shown in the legend, the rated operation point $(\Theta=11)$, a point with maximum indicator of divergence $(\boldsymbol{\Theta}=15)$ and two points with small indicator of divergence $(\boldsymbol{\Theta}=4$ $\& 28)$. These were computed for reference in order to support the comparison of results.
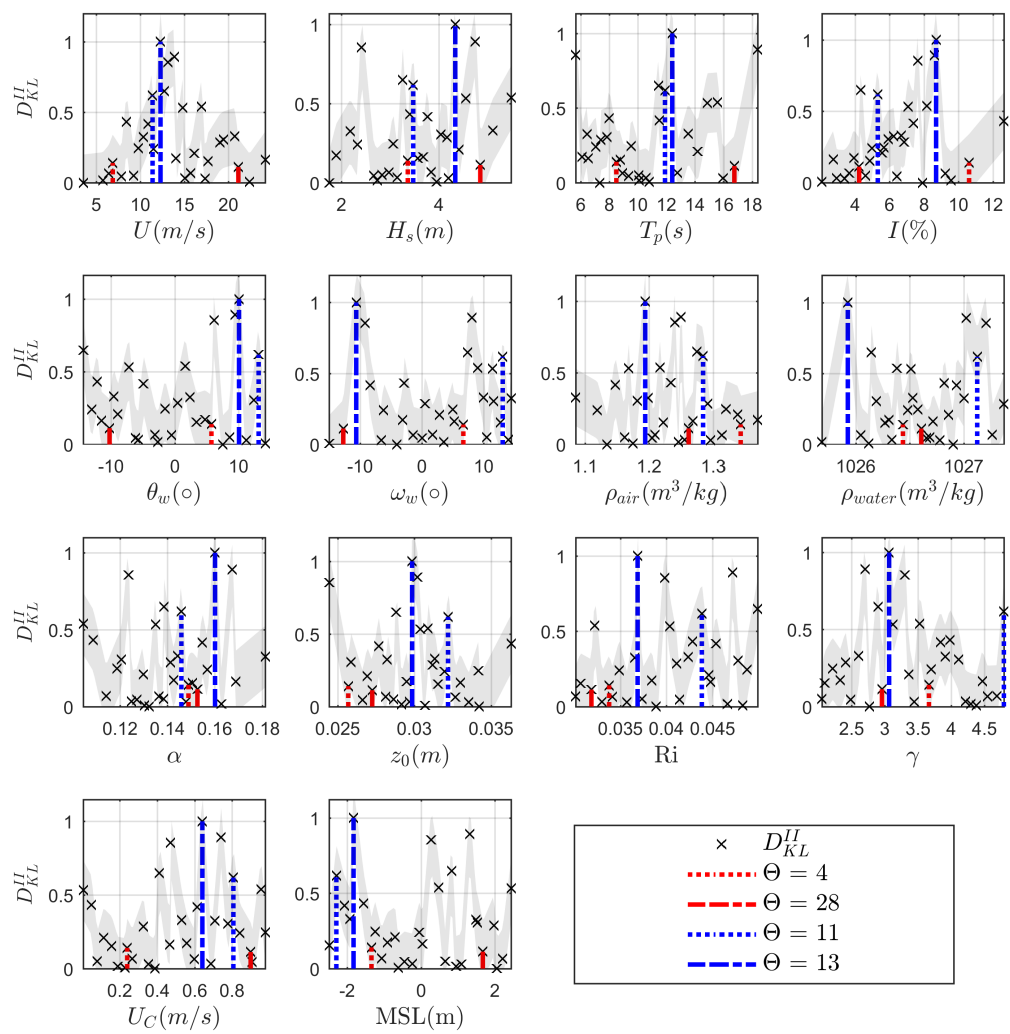

Figure 4: $D_{K L}^{I I}$ results for each individual $\theta_{i}$ variable. Gray highlighted only purpose is to facilitate the analysis of the results

The graphical analysis of the results shows one principal trend in the univariate data: $U$ as the variable in the DoE that presents a stronger indication of being the main contributor to the definition of the probabilistic behaviour of $D_{S H}$. Regarding changes of $U, D_{K L}^{I I}$ shows a peak close to the rated operation 
$(11.4 \mathrm{~m} / \mathrm{s})$. Operational states below the rated power have less contribution to the fatigue damage.

$I$ is also identified as an important variable in the DoE. There is asymmetry in $D_{K L}^{I I}$ in regard of changes in $I$. The gray shadow is used to facilitate the identification of these trends. The four points with largest values of $I$ are located below the rated $U$, explaining the descending trend after the value of $8.7 \%$.

$I$ and $U$ have a negative correlation and therefore large $U$ are not expected with large $I$. This is one of the reasons behind the position of the most sensitive points in the DoE, which are near the rated operation wind speed $(U=11.4 \mathrm{~m} / \mathrm{s})$. Nevertheless, if large $I$ occurs in combination with large $U$, such as in $\Theta=13$, the sensitivity indicator increases (in the present case maximum).

The transition to rated power has large influence in the results of $D_{S H}$. It is possible to infer that there is a peak in the sensitivity measure around the rated speed that occurs independently of the values that any of the remaining variables take. Cheng et al. (2003) showed that the largest loads in a pitch controlled turbine occur slightly above the rated power, and these loads are expected to be related to the increase in the fatigue damage when the OWT enters this operational region. In all the cases for similar values of $U$, large values of $I$ resulted in larger values of $D_{S H}$. Above and below the rated power, changes in $D_{K L}^{I I}$ are mainly controlled by these two variables. By studying the physical description of the system it is possible to understand that, while $U$ is the main variable in the calculation of the average thrust moment in the OWT, $I$ influences how the thrust fluctuates with time. Large $I$ is more likely to occur with low $U$, where the thrust and consequently moments are lower. Despite the increase in the Gaussian variability of $U$ caused by the large $I$, the loading cycles are expected to be less demanding.

The wave variables do not present any trend indicating a significant influence in the $D_{S H}$ probabilistic behaviour. For $H_{s}, T_{p}, \omega_{w}, \rho_{\text {water }}, \lambda$ and MSL a case where the variable has large and small values with high and low $D_{K L}^{I I}$ can be identified, see blue and red trimmed lines. The same occurs for $U_{C}$. Moreover, for these variables, the difference in the divergence from $\Theta=18$, an energetic sea state that occurs with the maximum positive variation of MSL, and $\boldsymbol{\Theta}=$ 28 , a very similar sea state and variation of MSL, indicates that the response is being dominated by the wind variables. Teixeira et al. (2017) highlighted before the low contribution of the wave variables to the tower structural fatigue in a pre-assessment of the DoE for OWT turbines. Despite the high amount of energy that these carry, common operational waves do not directly interact and load the tower component.

In the present case it is known beforehand that $\phi_{w}$ and $\omega_{w}$ present physical symmetry with respect to the OWT. Accordingly to the results presented $\phi_{w}$ does not seem to have major influence in the output distribution. $\Theta=12$ and 13, 23 and 28, are opposite symmetric where 12 and 23 represent a change in $D_{K L}^{I I}$ more than $100 \%$ bigger than 23 and 28 . The IEC standard foresees sudden $\phi_{w}$ change as one of the design load cases for which a OWT needs to be certified, which is the case where $\phi_{w}$ is expected to be have more influence. 
Accordingly to the ranges studied, $z_{0}, \alpha$ and $R i, \rho_{\text {air }}$ despite being directly related to the wind, do not show the same influence as $U$ and $I$. Both the largest of values $\rho_{\text {air }}$, second largest of $z_{0}$, and minimum of $R i$, which would be expected to contribute to increase $D_{S H}$, result in low damage operational states. For $\alpha$ the results are more evident in Figure 5.

One of the main purposes of using a single indicator, such as $D_{K L}^{I I}$ and a minimum damaging point for reference is to map the probabilistic field using the P-GSA results in operation maps, see Figure 5. This representation of the results is interesting for dynamic systems as it allows the DoE field analysis using a sensitivity and at the same time a design basis perspective.

If with the previous figure it was challenging to be certain of the influence of $\alpha$ or $\gamma$, in the map representation these seem to have limited effect in the tower fatigue. In 8 ) and 11) it can be seen that the values of $D_{S H}$ are mainly occurring due to coupling with $U$. The map representation 3) presents the most asymmetric behaviour in $D_{K L}^{I I}$. The largest values of $D_{K L}^{I I}$ occur near the rated speed, and then there is a gradual increase of the indicator value with the increase in $I$, for similar values of $U$. 

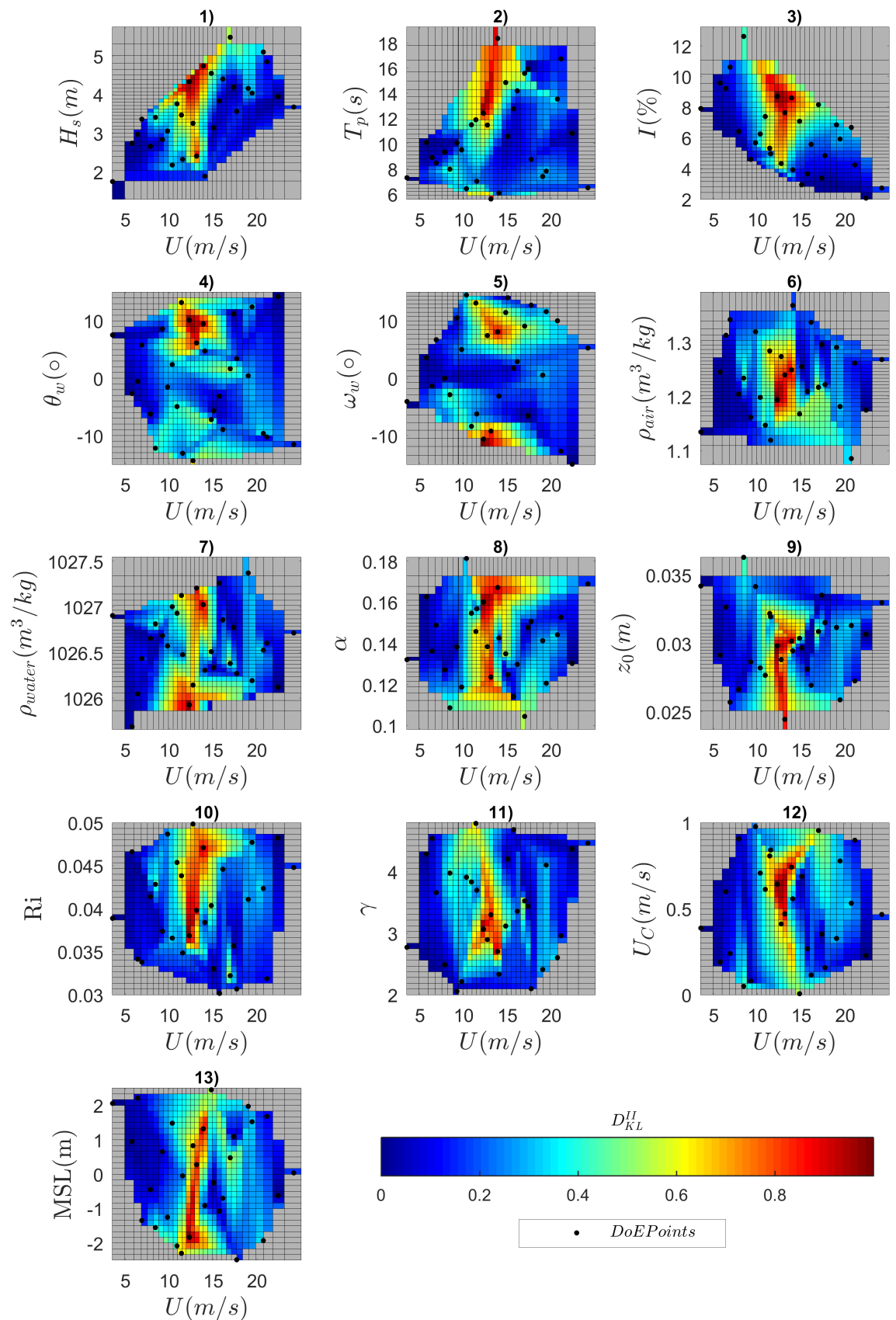

Figure 5: G-PSA mapping of fatigue damage operation for all the space of variables simulated (grey areas represent areas not covered by the LHS DoE).

The low influence of the wave variables is better identified in the operational 
maps. The areas of operation where $D_{K L}^{I I}$ is maximum seem to be imposed by the values of $I$ and $U$ instead of the wave parameters. Furthermore, one of the most energetic environmental wave conditions, $\Theta=26$, results in a smaller change in $D_{K L}^{I I}$ as the less energetic sea state, $\Theta=17$ (see Table 2). It is interesting to analyse the results relating to $\omega_{w}$ for Figures 4 and 5. According to the symmetry, the maps indicate that $\omega_{w}$ may have influence on the results. This is not supported by the other wave variable results, and therefore, may demand for more operational points to be assessed. These may be easily selected using this representation.

As $U$ shows important influence in the $D_{K L}^{I I}$, it can be seen that in all the maps it dominates the location of the most sensitive, thus, damaging points. The only exception is for $I$, where the most damaging points are also located at large $I$ values. A similar trend may appear to exist for $H_{s}$. However, in the case of $H_{s}$, considering $\boldsymbol{\Theta}=15$, and the pairing of $I$ and $H_{s}$ in $\boldsymbol{\Theta}=18$ and 22 , there is some indication that this may be related to $U$ and $I$. Furthermore, the relative dynamics of the tower induced movement due to the coupling of the tower and foundation are small, and operation is far from the structure resonant frequencies.

In a case of highly correlated variables, such as $U, H_{s}$ and $T_{p}$, the analysis of sensitivity needs to be taken meticulous because of the coupling between variables. Due to the effect of the correlation some of the grey areas are quite significant in the plots, e.g., plots a 1) and 3). In the context of fatigue, as a longterm event, most of the grey areas are very unlikely to be of interest. Therefore, they should be analysed in a design basis only if relevant information about the problem of fatigue may be held there. This may be the case of large $I$ and $U$ values.

Application of the KL to discrete datasets or using large number of evaluation points is expected to increase the complexity of the procedure. Hence, there is consequent increase of the numerical and computational efforts required to perform it. In such cases it may be of interest to use an approximation to continuous functions, or to interpolate from a predefined $D_{K L}$ map.

The whole probability characterization of the output is compiled in a single variable, which characterizes $D_{S H}$ uncertainty. The mapping can be one of the tools used to decide on a multi-dimensional analysis what additional points need to be addressed in order to have robust characterization results that sustain the design decisions. The case of $\omega_{w}$ is representative on how these maps may help to select new points in the analysis. It is difficult by analysing Figure 4 or Table 2 to select new points to extend the analysis since these do not allow to perceive the DoE space.

In some particular cases, the application of the KL may be limited. In the case of non-continuity in the compared distributions (e.g., non-overlaping distributions), the computation of KL may be challenging. Since the probabilistic behaviour of $D_{S H}$ was known, continuous models were used to compute the KL. Approximation to a continuous distribution, with quantification of the error, may be a robust approach to model the KL indicator. Smoothing techniques (Chen and Goodman, 1999) or heuristic approaches are often used as an 
alternative to deal with non-continuity in probability distributions and, hence, KL calculations. In such cases, attention should be given to the potential bias resulting from their application.

\section{Conclusions}

In the present work a Global Probabilistic Sensitivity Analysis was developed to analyse structural fatigue sensitivity of an OWT tower. Sometimes disregarded in the field of probabilistic analysis, global sensitivity analysis are powerful methodologies to increase the comprehension about the operation of a system.

Global sensitivity analysis in a probabilistic framework allows the characterization of the system's operation. In parallel, these allow the identification of important influences that the input variables may have in the probabilistic complexity of the system's response.

When considering an engineering perspective, there are cases where the measures used to compare probability distributions are of difficult interpretation, which may lead to an erroneous interpretation of the results. This fact is particularly important in reliability problems where the physical meaning of the results should be considered. In this context, a transformation was proposed to the Kullback-Leibler divergence measure in order to make it more suitable for application in a probabilistic global sensitivity analysis of reliability applications. The proposed transformation makes the Kullback-Leibler divergence completely symmetric and monotonic in different domains of interest in order to reflect symmetrically the sensitivity of the results. Nevertheless, only a reference probability distribution is required to define the full-field transformation proposed.

The application of a measure of divergence between two statistical distributions allows all the information about the probabilistic sensitivity to be compiled in a single indicator that carries information about the probability or uncertainty of the quantity studied.

Fourteen independent and dependent environmental variables that load the OWT were studied within a probabilistic reference example. A sampling technique that respects the statistical interaction between variables was implemented to cover the space of variables.

The global sensitivity results showed that the wind speed and turbulence intensity are the most influential variables for the probabilistic short-term damage rates of the tower. A meticulous analysis of these variables should be always considered in the tower analysis. Both variables are correlated, and their analysis cannot be disassociated. Saltelli et al. (2008) mentioned that is not unusual that, in a high dimensional space, only few parameters show influence in the output results.

Further works may consider adding another level of sensitivity to the approach, such as a local sensitivity estimate (e.g., one-factor-at-time analysis). To highlight that for a probabilistic based work, the amount of additional effort to do this may be unbearable. Therefore, a balanced approach should be 
considered. Given that fatigue analysis is a cumulative event where multiple small contributions sum up to define an indicator such as the one here defined, high non-linear local effects or discontinuities are not likely to happen in the different maps. In a practical basis, the current methodology should then be implemented with a refinement strategy focusing in; global operation and then in local areas that showed relevant sensitivities or that were not adequately covered by the DoE used to characterize the full-field operation.

\section{Acknowledgements}

This project has received funding from the European Union Horizon 2020 research and innovation programme under the Marie Sklodowska-Curie grant agreement No. 642453. 


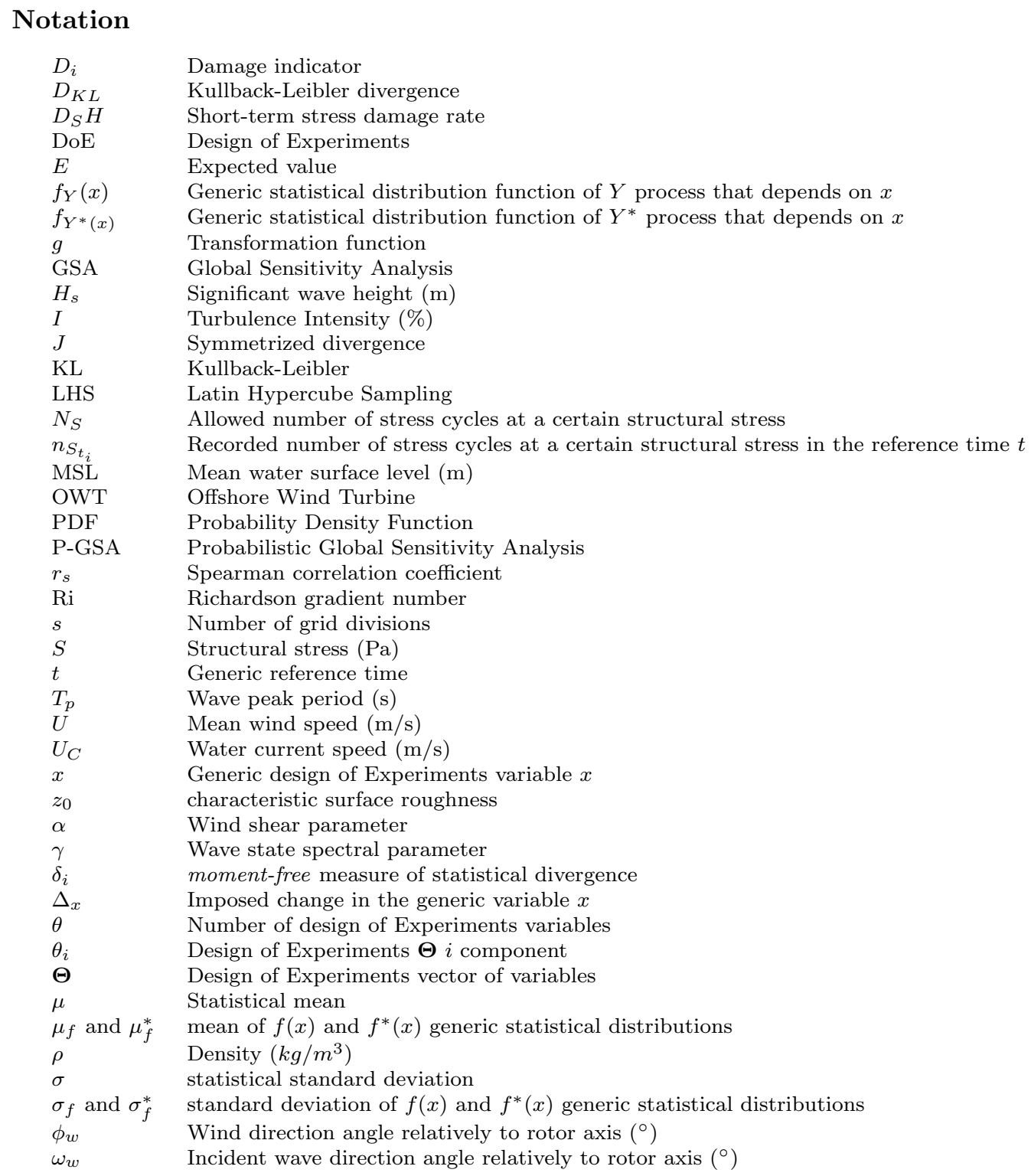

\section{References}

Balesdent, M., Morio, J., Marzat, J., 2013. Kriging-based adaptive importance sampling algorithms for rare event estimation. Structural Safety 44, 1-10.

Borgonovo, E., 2007. A new uncertainty importance measure. Reliability Engineering \& System Safety 92 (6), 771-784.

Caniou, Y., 2012. Global sensitivity analysis for nested and multiscale modelling. Ph.D. thesis, Université Blaise Pascal-Clermont-Ferrand II. 
Chen, S. F., Goodman, J., 1999. An empirical study of smoothing techniques for language modeling. Computer Speech \& Language 13 (4), 359-394.

Cheng, P., van Bussel, G., van Kuik, G., Vugts, J., 2003. Reliability-based design methods to determine the extreme response distribution of offshore wind turbines. Wind Energy 6 (1), $1-22$.

DNV, 2014. Design of offshore wind turbine structures. Offshore Standard DNV-OS-J101, DNV GL AS.

Efron, B., Stein, C., 1981. The jackknife estimate of variance. The Annals of Statistics, 586596.

Fatemi, A., Yang, L., 1998. Cumulative fatigue damage and life prediction theories: a survey of the state of the art for homogeneous materials. International journal of fatigue 20 (1), $9-34$.

Greegar, G., Manohar, C., 2016. Global response sensitivity analysis of uncertain structures. Structural Safety 58, 94-104.

Hayman, G., 2012. Mlife theory manual for version 1.00. Tech. rep.

Helton, J. C., Davis, F., Johnson, J. D., 2005. A comparison of uncertainty and sensitivity analysis results obtained with random and latin hypercube sampling. Reliability Engineering \& System Safety 89 (3), 305-330.

Helton, J. C., Davis, F. J., 2003. Latin hypercube sampling and the propagation of uncertainty in analyses of complex systems. Reliability Engineering \& System Safety 81 (1), 23-69.

Hoseyni, S. M., Di Maio, F., Vagnoli, M., Zio, E., Pourgol-Mohammad, M., 2015. A bayesian ensemble of sensitivity measures for severe accident modeling. Nuclear Engineering and Design 295, 182-191.

IEC, 2005. Wind turbines- part 1: Design requirements. Tech. Rep. 61400-1, International Electrotechnical Commission, Geneva, Switzerland.

Iman, R. L., Conover, W.-J., 1982. A distribution-free approach to inducing rank correlation among input variables. Communications in Statistics-Simulation and Computation 11 (3), $311-334$.

Iman, R. L., Helton, J. C., 1985. Comparison of uncertainty and sensitivity analysis techniques for computer models. Tech. rep., Sandia National Labs., Albuquerque, NM (USA).

Jonkman, B. J., 2009. Turbsim user's guide: Version 1.50.

Jonkman, J., Butterfield, S., Musial, W., Scott, G., 2009. Definition of a 5-mw reference wind turbine for offshore system development. National Renewable Energy Laboratory, Golden, CO, Technical Report No. NREL/TP-500-38060.

Kullback, S., 1997. Information theory and statistics. Courier Corporation.

Kullback, S., Leibler, R. A., 1951. On information and sufficiency. The annals of mathematical statistics $22(1), 79-86$.

Liu, H., Chen, W., Sudjianto, A., 2006. Relative entropy based method for probabilistic sensitivity analysis in engineering design. Journal of Mechanical Design 128 (2), 326-336.

Manache, G., Melching, C. S., 2008. Identification of reliable regression-and correlation-based sensitivity measures for importance ranking of water-quality model parameters. Environmental Modelling \& Software 23 (5), 549-562. 
Mesmacque, G., Garcia, S., Amrouche, A., Rubio-Gonzalez, C., 2005. Sequential law in multiaxial fatigue, a new damage indicator. International Journal of Fatigue 27 (4), 461-467.

Morris, M. D., Mitchell, T. J., 1995. Exploratory designs for computational experiments. Journal of statistical planning and inference 43 (3), 381-402.

Olsson, A., Sandberg, G., Dahlblom, O., 2003. On latin hypercube sampling for structural reliability analysis. Structural safety 25 (1), 47-68.

Park, C. K., Ahn, K.-I., 1994. A new approach for measuring uncertainty importance and distributional sensitivity in probabilistic safety assessment. Reliability Engineering \& System Safety 46 (3), 253-261.

Rabitz, H., Aliş, Ö. F., Shorter, J., Shim, K., 1999. Efficient input-output model representations. Computer Physics Communications 117 (1-2), 11-20.

Rachev, S. T., 1991. Probability metrics and the stability of stochastic models. Vol. 269. John Wiley \& Son Ltd.

Saltelli, A., Annoni, P., Azzini, I., Campolongo, F., Ratto, M., Tarantola, S., 2010. Variance based sensitivity analysis of model output. design and estimator for the total sensitivity index. Computer Physics Communications 181 (2), 259-270.

Saltelli, A., Ratto, M., Andres, T., Campolongo, F., Cariboni, J., Gatelli, D., Saisana, M., Tarantola, S., 2008. Global sensitivity analysis: the primer. John Wiley \& Sons.

Saltelli, A., Tarantola, S., Campolongo, F., et al., 2000. Sensitivity anaysis as an ingredient of modeling. Statistical Science 15 (4), 377-395.

Sobol, I. M., 1993. Sensitivity estimates for nonlinear mathematical models. Mathematical Modelling and Computational Experiments 1 (4), 407-414.

Sobol, I. M., 2001. Global sensitivity indices for nonlinear mathematical models and their monte carlo estimates. Mathematics and computers in simulation 55 (1-3), 271-280.

Stein, M., 1987. Large sample properties of simulations using latin hypercube sampling. Technometrics 29 (2), 143-151.

Sutherland, H. J., 1999. On the fatigue analysis of wind turbines. Tech. rep., Sandia National Labs., Albuquerque, NM (US); Sandia National Labs., Livermore, CA (US).

Teixeira, R., Nogal, M., O'Connor, A., 2018. On the suitability of the generalized pareto to model extreme waves. Journal of Hydraulic Research, 1-16.

Teixeira, R., O'Connor, A., Nogal, M., Krishnan, N., Nichols, J., 2017. Analysis of the design of experiments of offshore wind turbine fatigue reliability design with kriging surfaces. In: Procedia Structural Integrity. No. 5, 951-958.

Toft, H. S., Svenningsen, L., Sørensen, J. D., Moser, W., Thøgersen, M. L., 2016. Uncertainty in wind climate parameters and their influence on wind turbine fatigue loads. Renewable Energy 90, 352-361.

Türk, M., Emeis, S., 2010. The dependence of offshore turbulence intensity on wind speed. Journal of Wind Engineering and Industrial Aerodynamics 98 (8), 466-471.

Veldkamp, H. F., 2006. Chances in wind energy: a probalistic approach to wind turbine fatigue design.

Viana, F. A., Venter, G., Balabanov, V., 2010. An algorithm for fast optimal latin hypercube design of experiments. International journal for numerical methods in engineering 82 (2), $135-156$. 
Wirsching, P. H., Chen, Y.-N., 1988. Considerations of probability-based fatigue design for marine structures. Marine Structures 1 (1), 23-45.

Wu, W., Liou, H., Tse, H., 1997. Estimation of fatigue damage and fatigue life of components under random loading. International journal of pressure vessels and piping 72 (3), 243-249.

Yun, W., Lu, Z., Zhang, K., Jiang, X., 2017. An efficient sampling method for variance-based sensitivity analysis. Structural Safety $65,74-83$.

Zhang, K., Lu, Z., Cheng, L., Xu, F., 2015. A new framework of variance based global sensitivity analysis for models with correlated inputs. Structural Safety 55, 1-9. 